\title{
Treatment of hemophilia: a review of current advances and ongoing issues
}

\author{
This article was published in the following Dove Press journal: \\ Journal of Blood Medicine \\ 28 August 2010 \\ Number of times this article has been viewed
}

\section{Antonio Coppola \\ Mirko Di Capua \\ Matteo Nicola Dario \\ Di Minno \\ Mariagiovanna Di Palo \\ Emiliana Marrone \\ Paola leranò \\ Claudia Arturo \\ Antonella Tufano \\ Anna Maria Cerbone}

Regional Reference Center for Coagulation Disorders, Federico II University Hospital, Naples, Italy
Correspondence:Antonio Coppola

Regional Reference Center for

Coagulation Disorders, Federico

II University Hospital,Via S Pansini,

5-8013। Naples, Italy

$\mathrm{Tel}+390817462317$

Fax +3908I 5466152

Email antocopp@unina.it

\begin{abstract}
Replacement of the congenitally deficient factor VIII or IX through plasma-derived or recombinant concentrates is the mainstay of treatment for hemophilia. Concentrate infusions when hemorrhages occur typically in joint and muscles (on-demand treatment) is able to resolve bleeding, but does not prevent the progressive joint deterioration leading to crippling hemophilic arthropathy. Therefore, primary prophylaxis, ie, regular infusion of concentrates started after the first joint bleed and/or before the age of two years, is now recognized as first-line treatment in children with severe hemophilia. Secondary prophylaxis, whenever started, aims to avoid (or delay) the progression of arthropathy and improve patient quality of life. Interestingly, recent data suggest a role for early prophylaxis also in preventing development of inhibitors, the most serious complication of treatment in hemophilia, in which multiple genetic and environmental factors may be involved. Treatment of bleeds in patients with inhibitors requires bypassing agents (activated prothrombin complex concentrates, recombinant factor VIIa). However, eradication of inhibitors by induction of immune tolerance should be the first choice for patients with recent onset inhibitors. The wide availability of safe factor concentrates and programs for comprehensive care has now resulted in highly satisfactory treatment of hemophilia patients in developed countries. Unfortunately, this is not true for more than two-thirds of persons with hemophilia, who live in developing countries.
\end{abstract}

Keywords: bleeding, comprehensive care, clotting factor concentrates, hemophilia, inhibitors, prophylaxis, treatment

\section{Introduction}

Hemophilia A and B are congenital bleeding disorders caused by a deficiency or complete absence of coagulation factor VIII (FVIII) or factor IX (FIX), respectively. These X-linked disorders represent the large majority of inherited deficiencies of clotting factors, occurring in approximately one per 5000 and one per 50,000 male births, with no racial predilection. ${ }^{1}$ According to their residual endogenous FVIII/FIX concentrations, individuals with a factor level $<1 \mathrm{IU} / \mathrm{dL}$ are classified as severe hemophiliacs and represent about half of diagnosed cases. Subjects with factor levels between 1-5 IU/dL and $>5 \mathrm{IU} / \mathrm{dL}$ have moderate and mild hemophilia, respectively. Although the bleeding phenotype may be rather heterogeneous, even in severe hemophiliacs, ${ }^{2}$ this classification reflects the severity of clinical symptoms, with spontaneous joint and muscle bleeds being largely confined to patients with severe hemophilia.

Hemophilia A and B are difficult to distinguish from a clinical point of view. Replacement of hemostatic concentrations of the deficient factor is the mainstay of treatment for bleeding episodes, according to the type and severity of bleeds and until complete resolution of symptoms (Table 1). ${ }^{3}$ Recurrent joint bleeds, inevitably leading 
Table I Hemostatic factor concentrate dosages for replacement treatment in hemophilia

\begin{tabular}{lll}
\hline Setting* & FVIII (IU/kg) & FIX (IU/kg) \\
\hline Mild/moderate joint or muscle bleed & $20-30$ & $20-40$ \\
$\begin{array}{l}\text { Severe joint or muscle bleed, } \\
\text { mucosal bleeding with anemia, }\end{array}$ & $30-50$ & $40-60$ \\
$\begin{array}{l}\text { moderate post-traumatic bleed } \\
\text { CNS bleeding, cranial trauma, }\end{array}$ & $50-100$ & $50-100$ \\
surgery prophylaxis & & \\
\hline
\end{tabular}

Notes: "Treatment should be administered until complete resolution of bleeding or wound healing in the case of surgery. ${ }^{* *}$ Administration of I IU/kg of factor concentrate is assumed to result in an approximately $2 \%$ increase of FVIII and a I\% increase of FIX levels. According to their half-life in the absence of inhibitory antibodies, FVIII and FIX concentrates are usually given every 12 and 24 hours, respectively.

Abbreviations: CNS, central nervous system; FVIII, factor VIII; FIX, factor IX.

to crippling arthropathy, ${ }^{4}$ were the hallmark of this disease before the 1970s, when plasma fractions containing FVIII or FIX were still not available. At that time, mortality for bleeding was very high, and the life expectancy of persons with hemophilia was much lower than that of the general population. ${ }^{5,6}$ The discovery of cryoprecipitate in 1964 and the subsequent development of lyophilized factor concentrates in the 1970s paved the way for modern, effective hemophilia replacement therapy, providing the possibility of home therapy and enabling severe hemophiliacs to treat joint bleeds as early as possible. This was associated with a significant reduction in morbidity and the prospect of a more normal lifestyle for patients and their families. ${ }^{5}$ Subsequent to widespread blood-borne virus transmission in the late 1970s and early 1980s caused by the use of pooled plasma in the manufacture of factor concentrates, the need for improved safety of treatment became crucial for the hemophilia community. As a result, viral inactivation techniques for the production of plasma-derived factor concentrates were implemented. However, the most important advance was recombinant gene technology and protein purification techniques, which enabled the development of highly purified recombinant FVIII (rFVIII) and FIX products. ${ }^{5,7}$ The viral safety of factor concentrates dramatically improved the treatment and quality of life of hemophilia patients and significantly contributed to the increased use of regimens of regular infusion of factor concentrates to prevent bleeding and resulting joint damage (primary prophylaxis) and of home treatment. Thus, with viral safety now less of an issue and with the more widespread use of prophylaxis to prevent arthropathy, the most serious and challenging complication of treatment, mainly in hemophilia A, there remains the risk of developing inhibitory alloantibodies. ${ }^{5,8}$

Patients with hemophilia may now receive excellent treatment, based on very safe antihemophilic products and effective approaches for comprehensive care, and achieve an excellent quality of life. Unfortunately, this is true only for patients living in developed countries, in which a wide variety of factor concentrates are available, as shown in Table 2. The aim of this review is to address current advances and clinical strategies for treatment of hemophilia, also focusing on ongoing issues and perspectives in this setting.

Table 2 Type of products currently used for replacement therapy in hemophilia A and B

\begin{tabular}{|c|c|}
\hline Type of product & Comments \\
\hline \multicolumn{2}{|l|}{ Hemophilia A } \\
\hline Intermediate-purity plasma-derived FVIII concentrates & $\begin{array}{l}\text { Purification from cryoprecipitate through multiple precipitation; } \\
\text { single-step viral inactivation }\end{array}$ \\
\hline High-purity plasma-derived FVIII concentrates & $\begin{array}{l}\text { Purification through ion-exchange, heparin ligand or monoclonal } \\
\text { antibody chromatography; single- or double-step viral inactivation }\end{array}$ \\
\hline Full-length recombinant FVIII concentrates & $\begin{array}{l}\text { - From BHK-cultured cells in the presence of HSA, stabilized } \\
\text { in sucrose; SD viral inactivation } \\
\text { - From CHO-cultured cells without HSA, stabilized in } \\
\text { trehalose; SD viral inactivation }\end{array}$ \\
\hline B-domain deleted recombinant FVIII & From CHO-cultured cells without HSA and animal protein; \\
\hline concentrate & SD viral inactivation and nanofiltration \\
\hline \multicolumn{2}{|l|}{ Hemophilia B } \\
\hline High-purity plasma-derived FIX concentrates & $\begin{array}{l}\text { Purification through immunoaffinity or ion exchange plus carbohydrate- } \\
\text { or heparin-ligand chromatography; single- or double-step viral inactivation }\end{array}$ \\
\hline Recombinant FIX concentrate & From CHO-cultured cells, without HSA; nanofiltration \\
\hline \multicolumn{2}{|l|}{ Hemophilia with inhibitors (by-passing agents) } \\
\hline APCC & $\begin{array}{l}\text { Plasma-derived; batch-controlled surface activation } \\
\text { of prothrombin complex; vapour heat viral inactivation }\end{array}$ \\
\hline rFVIla & $\begin{array}{l}\text { From BHK cultured cell; FVII autoactivation during } \\
\text { chromatographic purification; SD viral inactivation }\end{array}$ \\
\hline
\end{tabular}

Abbreviations: BHK, baby hamster kidney; CHO, chinese hamster ovary; HSA, human serum albumin; SD, solvent/detergent; FVIII, factor VIII; FIX, factor IX; APCC, activated prothrombin complex concentrates; rFVIla, recombinant activated factor VII. 


\section{Prophylaxis versus on-demand replacement therapy}

If hemophilia is already known to be in the family, the disorder is usually diagnosed before the occurrence of significant bleeding, ie, in the neonatal period or earlier by prenatal diagnostic methods. ${ }^{9}$ In sporadic cases (first diagnosis in a family, approximately $30 \%-40 \%$ of cases), the severe patients are diagnosed before the age of one year because of mucosal or soft tissue hemorrhages, usually after trauma. ${ }^{10}$ However, an incidence of intracranial or extracranial hemorrhage at delivery of $3.6 \%$ has been calculated. ${ }^{11}$ With the exception of these and of patients requiring surgery, replacement treatment in hemophilic children is usually started at the time of the first joint bleed, occurring in most patients before the age of two years, together with joint mobilization and load. ${ }^{2}$ Ankles, and later knees and elbows, are most frequently affected. However, on-demand factor infusion, which is the treatment in response to an acute bleeding episode, is able to stop the hemorrhage, but does not impact the presence of blood already accumulated in the affected joint and its deleterious effects on synovial tissues. ${ }^{4}$ Deposition of iron is believed to trigger an inflammatory reaction, release of oxidative products, and vascular proliferation. ${ }^{4}$ Synovial hyperemia and hypertrophy facilitates repeated bleeding episodes ("target joint") and amplification of phenomena leading, in a vicious cycle, to chronic arthritis, with progressive damage of cartilage and bone. Degenerative joint disease (hemophilic arthropathy) results in decreased range of motion, functional impairment, and chronic pain. This is associated with reduction of physical fitness and muscle atrophy, and in turn, exacerbation of functional joint impairment and tendency to bleeding.

Although individual patterns of bleeding vary substantially, even in patients with severe FVIII/FIX deficiencies, ${ }^{2,12}$ most patients experience $10-15$ joint bleeds per year. ${ }^{13}$ Clinical and radiologic follow-up of children with severe hemophilia has shown that progressive joint degeneration can be documented in more than half of patients within six years from the first bleeding episode, often associated with restriction of activity. ${ }^{13}$ The severity of joint disease and dysfunction is closely related to the frequency of bleeding episodes, given that a direct relationship between the clinical or radiologic scores describing the severity of arthropathy ${ }^{14,15}$ and the cumulative number of hemarthroses has been clearly shown. ${ }^{13,16}$

The recognition that patients with moderate or mild hemophilia (who have FVIII/FIX levels $>1 \%$ ) show a low frequency of joint bleeds and rarely develop severe arthropathy ${ }^{17}$ led to definition of the pathophysiologic background and pioneer prophylaxis regimens in Sweden. The aim of treatment was (and still is) to minimize the number of joint bleeds from an early age by converting the severe form of hemophilia to a milder form, in order to prevent or reduce musculoskeletal impairment from hemophilic arthropathy. Prophylaxis in hemophilia is defined as the infusion of factor replacement concentrates in order to prevent bleeding, and usually refers to a regular, continuous long-term regimen of treatment, as shown in Table 3. The results of the Swedish ${ }^{18,19}$ and of subsequent, retrospective, uncontrolled studies ${ }^{16,20-23}$ documented the clinical and social benefits of different prophylaxis regimens, in terms of reduction of frequency of total and joint bleeds and, in particular, on long-term clinical outcome in terms of arthropathy, assessed by clinical and radiologic scores, and of patient quality of life, as reported in Table 4. These studies also showed that the earlier the start of prophylaxis, the better the results for patient joint status. These findings led to the current definitions of prophylaxis, ${ }^{24,25}$ which is focused on the prevention of joint abnormalities, in order to enable normal life and psychosocial development for hemophilic children, including the possibility of physical activities, regular school attendance and, consequently, social and work opportunities. Even though the number of joint bleeds resulting in irreversible joint damage

Table 3 Definitions of replacement treatment regimens in hemophilia

\begin{tabular}{|c|c|}
\hline Regimen & Definition \\
\hline Primary prophylaxis A & $\begin{array}{l}\text { Long-term continuous }{ }^{*} \text { treatment started after the first joint bleed } \\
\text { and before the age of two years }\end{array}$ \\
\hline Primary prophylaxis B & $\begin{array}{l}\text { Long-term continuous }{ }^{*} \text { treatment started before the age of two years, } \\
\text { in the absence of clinically evident joint bleeds }\end{array}$ \\
\hline Secondary prophylaxis A & $\begin{array}{l}\text { Long-term continuous }{ }^{*} \text { treatment not fulfilling the criteria for primary } \\
\text { prophylaxis, ie, started after two or more joint bleeds or at age }>\text { two years }\end{array}$ \\
\hline Secondary prophylaxis B (short-term prophylaxis) & $\begin{array}{l}\text { Intermittent regular (short-term) treatment, generally started } \\
\text { because of frequent bleeds }\end{array}$ \\
\hline On-demand or episodic therapy & Treatment given when bleeding occurs \\
\hline
\end{tabular}

Note: *At least 46 weeks/year, with the aim of treating 52 weeks/year up to adulthood. 
Table 4 Documented clinical benefits and barriers to implementation of prophylaxis in children with hemophilia

\begin{tabular}{lc}
\hline Clinical advantages & Barriers \\
\hline - Reduction of severity (prevention of life-threatening & - Costs of coagulation factor concentrates \\
hemorrhages) and of frequency of total and joint bleeds & (prohibitive in developing countries) \\
- Prevention/reduction of joint and muscle impairment & - Inadequate peripheral venous access in children \\
(hemophilic arthropathy) $\rightarrow$ reduction of physical restrictions & (need for central venous access devices, more \\
and ability to participate in physical activities and sports & recently arteriovenous fistulae) \\
- Reduction of hospitalizations, professional visits & - Acceptance of treatment (poor perceived need \\
and examinations & and knowledge of benefits, heterogeneity \\
- Reduction of patients'/caregivers' school/work days lost $\rightarrow$ regular school & of bleeding phenotype) \\
attendance and better achievement $\rightarrow$ better work and social opportunities & - Home treatment not possible \\
- Reduction of psychologic impairment and higher levels of quality of life & - Logistic problems (difficult access \\
- Prevention of development of inhibitors (to be further evaluated) & to hemophilia treatment centers)
\end{tabular}

is still unknown, ${ }^{4}$ recent revisions by the European Pediatric Network for Haemophilia Management group ${ }^{25}$ now define primary prophylaxis as regular long-term regimens started before the age of two years, after the first joint bleed (A), or in the absence of clinically evident joint bleeds (B). Treatment started after the age of two years or after two or more joint bleeds is considered to be secondary prophylaxis, and is aimed at avoiding (or delaying) the progression of joint damage. ${ }^{25,26}$

The lack of randomized controlled trials comparing prophylaxis versus on-demand treatment in hemophilic children was highlighted by a Cochrane review in 2005. ${ }^{27}$ Recently two studies carried out with rFVIII products addressed this issue. The Joint Outcome Study (JOS) was the first published, prospective, randomized trial comparing prophylaxis versus on-demand treatment. ${ }^{28}$ The primary endpoint of the study was the prevention of joint deterioration by prophylaxis, started prior to or at the time of the second joint bleed (between six and 30 months), and assessed at the age of six years by radiography and/or by the most advanced approach for evaluating joint structure, ie, magnetic resonance imaging (MRI). ${ }^{29}$ Beyond the expected impact on number of bleeds, including life-threatening hemorrhages, the JOS study provided evidence of an approximately six-fold reduction of risk of joint damage in 32 children on prophylaxis with rFVIII 25 IU/kg every other day compared with 33 patients receiving intensive on-demand treatment (40 IU/kg, then $20 \mathrm{IU} / \mathrm{kg}$ at 24 and 72 hours and every other day until recovery). Interestingly, only the MRI assessment showed a significant difference in joint outcome, with more than half the joint abnormalities being not detectable by conventional radiologic evaluation. ${ }^{28}$ The JOS study confirmed that MRI is the preferable imaging technique in hemophilic children, also being able to reveal signs of joint deterioration in the absence of overt history of hemarthroses and of abnormalities at physical examination, although the interpretation and scoring of findings is still debated. ${ }^{29}$ Based on the results of the JOS study showing the efficacy of prophylaxis in preserving joint health and preventing life-threatening hemorrhages, the authors concluded that prophylaxis in hemophilic children is justified.

The results of another randomized trial carried out in Italy, the ESPRIT (Evaluation Study on Prophylaxis: a Randomized Italian Trial), have been recently published in abstract form. ${ }^{30}$ Forty patients aged younger than seven years (median two years) with negative clinical and radiologic scores at study entry were randomized to receive $\mathrm{rFVIII} 25 \mathrm{IU} / \mathrm{kg}$ three times weekly or on-demand treatment ( $\geq 25 \mathrm{IU} / \mathrm{kg}$ until complete healing). Significantly lower bleeding frequency and Petterson scores have been reported in children on prophylaxis than in those on on-demand treatment after a long-term follow-up of 10 years. The results of the JOS and ESPRIT studies definitely provided evidencebased recommendations for prophylaxis as the treatment of choice in hemophilic children, as recommended by the World Health Organization (WHO) and the World Federation of Hemophilia since $1994 .{ }^{31}$

Although there is general agreement among investigators that early initiation of prophylaxis (usually before two years of age) is ideal, there is still debate about the intensity of treatment regimens ${ }^{32}$ and how to initiate prophylaxis, as shown in Table 5. In this respect, barriers to early implementation of prophylaxis are the need for frequent venous access and adequate training of families for home treatment, in order to maximize adherence to such a highly demanding treatment, the perceived need for which and knowledge of benefits are often poor. ${ }^{33}$ These problems and the differences in patient bleeding patterns (approximately $10 \%$ of severe hemophiliacs are mild bleeders and variability in the age of 
Table 5 Prophylaxis regimens in hemophilia

\section{High-dose regimens (Sweden, Germany, UK, US, Italy) \\ Hemophilia A \\ $25-40 \mathrm{IU} / \mathrm{kg}$ \\ Escalating dose regimens}

Hemophilia B

$25-40 \mathrm{IU} / \mathrm{kg}$

Intermediate-dose regimens (The Netherlands)

Hemophilia A $\quad$ 15-25 IU/kg

Hemophilia B
$30-50 \mathrm{lU} / \mathrm{kg}$ three times weekly or every other day

$500 \mathrm{IU}$ once weekly, rapidly increased to twice and three times weekly on the basis of venous access (Sweden)

$50 \mathrm{IU} / \mathrm{kg}$ once weekly $\rightarrow 30 \mathrm{IU} / \mathrm{kg}$ twice weekly $\rightarrow 30 \mathrm{IU} / \mathrm{kg}$

every other day, according to bleeding frequency (Canada)

two or three times

two to three times weekly

once to twice weekly first joint bleed has been reported $)^{2,12}$ led to individualization in implemention of full-dose primary prophylaxis after an early start of treatment using step-up regimens. While the Swedish approach encompasses a brief temporary once-aweek infusion step, with the aim to escalate children quickly towards twice-weekly infusions and then to three infusions per week as full prophylaxis, based on the availability of adequate peripheral veins or, more rarely, on the bleeding frequency, ${ }^{34}$ the Canadian approach consists of a more gradual escalation based on the number of joint bleeds, comprising once-weekly infusions at a dose of $50 \mathrm{IU} / \mathrm{kg}$ started between one and two years of age, with a clinical follow-up every three months. Patients experiencing three bleeds in the same joint or four total bleeds over a three-month period then have an increase in their prophylaxis dose by infusion of $30 \mathrm{IU} / \mathrm{kg}$ twice weekly and then by a regimen of $25 \mathrm{IU} / \mathrm{kg}$ every other day. ${ }^{35}$ Interestingly, in the Canadian experience, $40 \%$ of children maintained the once-weekly infusion and $16 \%$ reached the full-dose prophylaxis regimen over a median 4.1-year follow-up. However, long-term follow-up studies of patients treated with such regimens are needed to evaluate the possible impact on joint outcome (the development of target joints in $22.5 \%$ of children has raised some concerns). ${ }^{35}$ Nevertheless, the gradual, escalating introduction of prophylaxis allows patients and families to accept peripheral venipunctures better psychologically, enables development of robust veins capable of being punctured more frequently, achieves independence for home treatment. Both the Swedish and Canadian experiences resulted in a significantly diminished need for central venous access devices (CVADs).

A major barrier to the widespread use of prophylaxis in hemophilic children, as shown in Table 4, remains the costs of clotting factor concentrates, which are particularly prohibitive in developing countries. ${ }^{33}$ The JOS study showed clearly that factor consumption for patients on regular prophylaxis is about 2.5-fold higher than that of episodic treatment. The annual cost of prophylaxis for a child weighing $50 \mathrm{~kg}$ was estimated to amount to $\$ 300,000 .^{28}$ However, on-demand treatment is still the predominant replacement approach in many developed countries. According to recent data, only $19 \%$ of children receive primary prophylaxis in the US, and a large variability is reported also in European countries, with highest figures (73\%) in Sweden. ${ }^{36}$ In this respect, the wider availability of safer rFVIII products was the determinant for large-scale implementation of primary prophylaxis in the 1990s in many countries outside Northern Europe. ${ }^{7}$

Finally, there are some other ongoing issues with regard to the duration of primary prophylaxis and delayed start of prophylaxis. Recent recommendations state that prophylaxis should be continued indefinitely, and that it is the treatment of choice for hemophiliacs at any age. ${ }^{37-39}$ Some groups of investigators have tried to evaluate variables affecting the phenotypic severity of the disease in order to identify those patients able to stop prophylactic treatment without adverse clinical effects. ${ }^{40,41}$ Fischer et $\mathrm{al}^{40}$ reported that about one-third of patients who discontinued prophylaxis permanently maintained a low number of joint bleeds. Similar findings were shown in a Dutch-Danish study. ${ }^{41}$ Thus, in order to predict which patients might be able to stop prophylaxis safely, a score based on age at start of prophylaxis, weekly dose of prophylaxis, and joint bleed frequency on prophylaxis has been proposed. ${ }^{41}$ However, more research is required to identify these patients and to determine whether discontinuing prophylaxis during adolescence or adulthood may compromise the positive joint outcome achieved.

As previously mentioned, due to the fact that in many countries primary prophylaxis was introduced more recently on a large scale, secondary prophylaxis has been started in many children of school age or later, in most cases because of a high bleeding frequency or after the development of target joints when patients were treated on-demand. There are a number of studies in the literature on the role of secondary prophylaxis in children, ${ }^{13,42-45}$ and more recently also for adult patients. ${ }^{46-48}$ On the whole, these studies show that 
even delayed prophylaxis is able to reduce the frequency of bleeding as well as patients' physical and psychologic restrictions. There are, however, uncertainties regarding the possible impact on the progression of arthropathy, particularly in adults. However, the improvement of quality of life appears to counterbalance the undoubtedly higher costs of secondary prophylaxis, also in adolescent and adult patients. ${ }^{49}$

\section{Prophylaxis, type of concentrate, and development of inhibitors}

Approximately $30 \%$ of patients with severe hemophilia A generate antibodies (inhibitors) against therapeutically administered FVIII, typically during the first 20-50 days of exposure..$^{50}$ These figures are lower in severe hemophilia B patients (about $5 \%$ ), although inhibitor prevalence is similar to that reported in hemophilia A in some populations. According to the highest documented inhibitor level and the presence of an anamnestic peak at factor concentrate re-exposure, high-responding $(>5$ $\mathrm{BU} / \mathrm{mL}$ ) or low-responding (always $<5 \mathrm{BU} / \mathrm{mL}$ ) inhibitors are distinguished. In a minority of patients, inhibitors are detected temporarily, and are no longer found during factor replacement treatment (transient inhibitors).

Development of inhibitors remains the most serious and challenging complication of treatment for hemophilia, because the safe and effective standard of care, particularly prophylaxis, is precluded. Despite improvements in strategies for treatment in this setting, ${ }^{51,52}$ patients with inhibitors experience higher levels of morbidity and mortality ${ }^{53,54}$ and a poorer quality of life related to their orthopedic status than patients who do not develop inhibitors. ${ }^{54,55}$ Moreover, the economic burden of this complication is the highest reported for a chronic disease. ${ }^{54}$

Inhibitors develop as a result of a complex interplay between many genetic and environmental factors (Figure 1). ${ }^{56}$ The role of the type of causative mutations in FVIII or FIX genes has been clearly recognized and is the basis for increased risk in patients with a family history of inhibitors. ${ }^{57}$ Concordance family studies show that factors other than FVIII/FIX gene mutations are involved. As a consequence of the higher clinical impact of inhibitors, most studies have been carried out in patients with hemophilia A. There is an emerging role for polymorphisms of immunoregulatory genes that may increase (interleukin-10 and tumour necrosis factor- $\alpha$ ) or reduce (CTLA-4) inhibitor risk, the heterogeneous ethnic distribution of which may correlate with the higher inhibitor risk in non-Caucasian patients. ${ }^{58-60}$ The role of nongenetic factors has been increasingly supported by evolving concepts of the immune response based on the "danger model". ${ }^{61}$ The immune system is activated by alarm signals from injured
GENETIC BACKGROUND

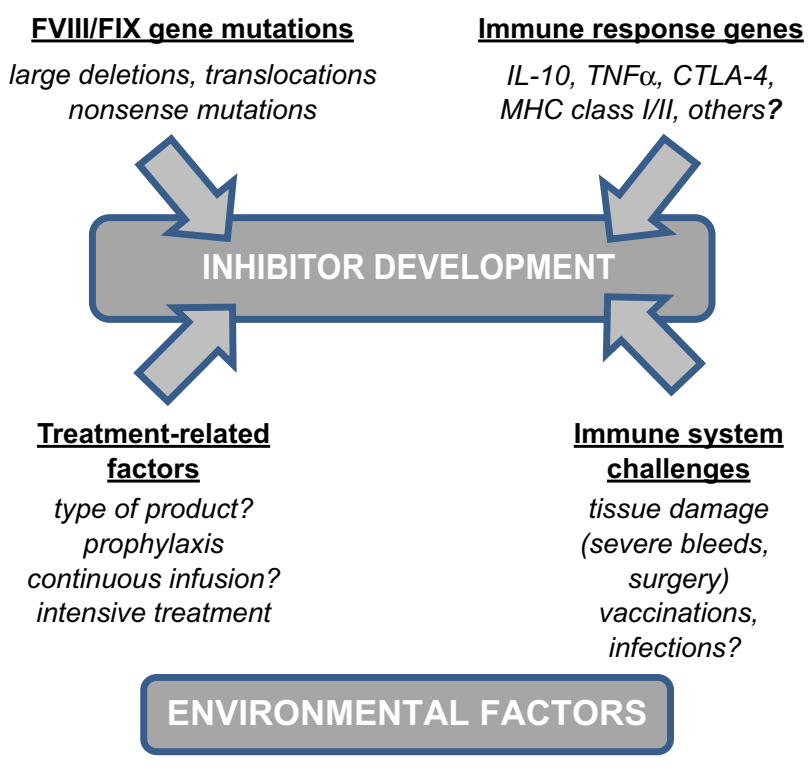

Figure I Factors contributing to inhibitor development in hemophilic patients. Multiple genetic and environmental factors interact during the first exposures to factor concentrate replacement treatment. Most data have been obtained from studies in hemophilia A patients, who develop inhibitors with higher frequency than those with hemophilia B.

tissues to a greater extent than by recognition of nonself. In this respect, the presentation of exogenous FVIII/FIX may not be sufficient for initiating an immune response. In the presence of dangerous conditions (ie, severe bleeds, or trauma or surgery with major tissue injury), the foreign protein is intensively presented (high-dose and/or prolonged treatment) in association with signals that upregulate the cellular T- and B-lymphocyte response. Conversely, regular exposure to lower doses of antigen, in the absence of danger signals, which occurs with regular prophylaxis, may allow the immune system to tolerate the foreign protein. Recent studies ${ }^{62,63}$ consistently report an increased inhibitor risk for patients receiving initial intensive treatments (surgery or severe bleeds requiring high-dose and/or prolonged treatment). On the other hand, after preliminary uncontrolled data were published, ${ }^{22,64}$ a protective effect against the development of inhibitors has been shown by multivariate analysis in a case-control Italian study, ${ }^{65}$ reporting a $70 \%$ reduction of inhibitor risk in children starting prophylaxis at a median age of 35 months compared with children receiving on-demand treatment. Similar findings were reported in the larger multinational Concerted Action on Neutralizing Antibodies in Severe Hemophilia A (CANAL) study, in which early regular prophylaxis (started at a median age of 20 months) was an independent predictor associated with a $60 \%$ lower risk 
of development of inhibitors than on-demand treatment. ${ }^{62}$ Starting with these pathophysiologic and clinical observations, inhibitor prevention strategies avoiding immunologic danger signals are being investigated. A German experience has recently been published. ${ }^{66} \mathrm{~A}$ "tolerization" regimen of $25 \mathrm{IU} / \mathrm{kg}$ FVIII once weekly was started early, as soon as a bleeding tendency was shown in soft tissues and muscles (at approximately 10 months of age). The frequency of infusion was increased to two or three times a week as required according to the bleeding tendency. Moreover, danger signals were minimized by avoidance of FVIII infusions during infections, on the same day as vaccinations, and delaying elective surgery during the first 20 days of exposure. Only one of 14 patients receiving this new regimen developed inhibitors compared with 14 of 30 retrospectively analyzed children treated with standard prophylaxis, resulting in a $>90 \%$ reduction of risk. ${ }^{66}$ Therefore, at least in hemophilia A, clinical approaches at the start of replacement treatment are expected to evolve towards prophylaxis regimens able to prevent development of inhibitors together with joint deterioration. The encouraging data for additional benefits of primary prophylaxis could further help to overcome barriers to the acceptance of this type of regimen that has undeniably transformed the lives of children with severe hemophilia and their families. ${ }^{49}$

The CANAL study also investigated the relationship between FVIII product type (ie, plasma-derived versus recombinant) and switching between FVIII products with regard to the risk of inhibitor development. ${ }^{67}$ Plasma-derived FVIII products were not associated with a lower risk of inhibitors than rFVIII products, nor did switching between FVIII product brands increase the risk of inhibitors. ${ }^{67}$ At variance with the comparison of the retrospective French cohorts showing lower inhibitor risk in patients treated with a plasmaderived product, ${ }^{68}$ the CANAL results were consistent with the findings of another recent English study which failed to detect significant differences in inhibitor risk by multivariate analysis, ${ }^{69}$ and highlighted that clinically relevant inhibitors develop with substantially comparable figures irrespective of type of product. On the other hand, the prospective long-term rFVIII registration studies clearly showed that about one-third of detected inhibitors were transient and that less than half were high-responding inhibitors $(>10 \mathrm{BU} / \mathrm{mL}) .{ }^{70}$ Therefore, possible discrepancies in inhibitor detection are likely to be due to the different study designs (retrospective, multicenter, and multinational, involving many product brands and modalities of treatment, with different exposure days) and, notably, to the fact that low-titer and transient inhibitors are usually missed in the older plasma-derived FVIII studies, thus resulting in underestimation of overall inhibitor incidence. On the whole, the hypothesized protective role of von Willebrand factor contained in plasma-derived concentrates in terms of reducing FVIII immunogenicity is presently supported more by in vitro data and preclinical experiments in animal models ${ }^{71}$ rather than by clinical data.

\section{Management of inhibitors in hemophilia}

Strategies for clinical management of patients with inhibitors are rather heterogeneous because of the variability of clinical manifestations and of responsiveness to available therapies and, in particular, the lack of rigorous studies providing enough high-level evidence to formulate treatment guidelines. The development of a specific inhibitor to FVIII or FIX results in partial or complete lack of efficacy of factor concentrates. In patients with transient or low-responding inhibitors, or with an actual low inhibitor titer $(<5 \mathrm{BU} /$ $\mathrm{mL}$ ), bleeding episodes may be managed by increased dosages of FVIII/FIX concentrates. However, in the majority of patients with high-responding inhibitors, bypassing agents (eg, recombinant activated factor VII, rFVIIa, and activated prothrombin complex concentrates, aPCC) are needed (Figure 2) and different regimens of treatment (dose, modality and frequency of administration) are reported. . $^{51,72,73}$ Moreover, recent anecdotal reports suggest improved efficacy with combined sequential use of rFVIIa and aPCC, and increasing data are being collected on prophylactic regimens with both agents for patients with life-threatening or very frequent bleeding. ${ }^{52,73}$ Given the serious clinical consequences of inhibitors, immune tolerance induction (ITI) by means of frequent and long-term administration of concentrates to

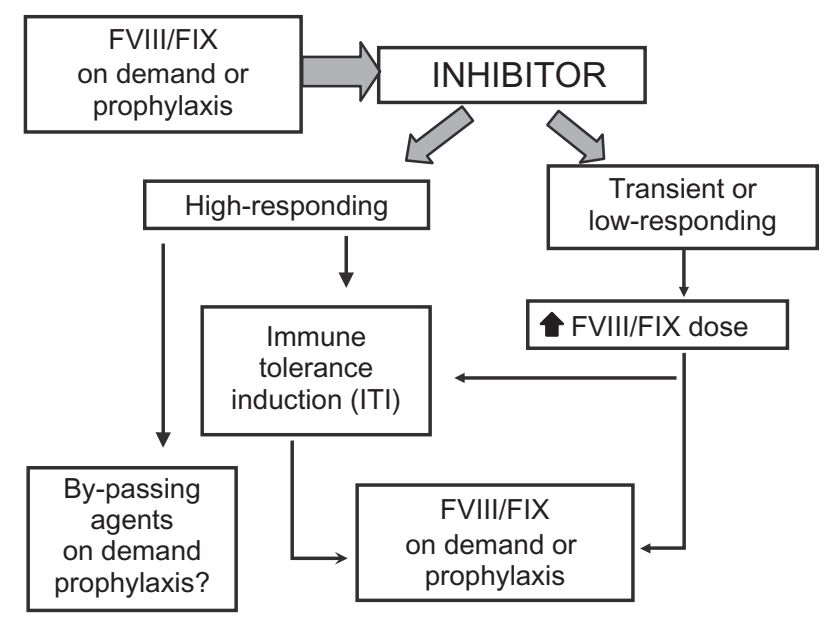

Figure 2 Current treatment strategies for patients with hemophilia who develop inhibitors. 
eradicate inhibitors and restore standard factor concentrate treatment and prophylaxis feasibility (Figure 2), is attempted in most patients as soon as possible after diagnosis of inhibitors, especially in children. ${ }^{72-76}$ As for risk factors of inhibitor development, the majority of studies have also been carried out in patients with hemophilia A, using a variety of therapeutic protocols (ie, dose and type of concentrate, interval of infusions, and immunomodulating agents). ${ }^{76-80}$ Data for hemophilia B are even more scarce because ITI is rarely attempted in these patients, with severe anaphylactic reactions and development of nephrotic syndrome having been reported. ${ }^{77}$

In the $1990 \mathrm{~s}$, retrospective data for a considerable number of patients who underwent ITI were collected in order to obtain more information about prognostic of ITI success. Most of the data comes from three registries, ie, the International Immune Tolerance Registry (IITR), ${ }^{78}$ the North American Immune Tolerance Registry (NAITR), ${ }^{79}$ and the German Immune Tolerance Registry, ${ }^{80}$ with success rates reported between $60 \%$ and $80 \%$. In a meta-analysis of the data from the IITR and the NAITR, published in 1999, among the variables analyzed (ie, historical inhibitor peak titer, inhibitor titer before ITI initiation, FVIII dosing, and FVIII products), only a historical inhibitor peak titer $<50 \mathrm{BU}$ and an inhibitor titer $<10$ BU immediately before ITI initiation were identified as predictors of ITI success. ${ }^{81}$ However, ITI registries had some limitations, ie, a retrospective design and heterogeneous patient and treatment characteristics, in particular the definition of endpoints. Recently, an Italian retrospective-prospective registry providing central revision of ITI outcome showed that, similar to inhibitor development, the type of FVIII gene mutations may predict ITI success. ${ }^{82}$ However, the optimal ITI regimen is still being debated. The International Immune Tolerance Induction Study, the first prospective, randomized, controlled trial of ITI in goodrisk patients, focusing on the dose issue $(200 \mathrm{IU} / \mathrm{kg} /$ day versus 50 IU/kg t.i.w.), ${ }^{83}$ and the Rescue Immune Tolerance (RESIST study), addressing the role of different types of FVIII concentrate in ITI rescue and now in its early phase, ${ }^{84}$ are expected to resolve some of the unanswered questions. With the aim of providing useful information for hemophilia caregivers, an international panel of opinion leaders developed consensus recommendations for ITI on the basis of the available published literature and the collective clinical experience of the panel. ${ }^{76}$ Rated according to the level of supporting evidence, the main consensus recommendations for the FVIII product type were that ITI is successful using FVIII products regardless of von Willebrand factor content, with no definitive data supporting the superiority of any FVIII product, and that most patients can effectively achieve tolerance with the same FVIII product in use at the time of inhibitor detection. For all these recommendations the level of evidence was IIb. ${ }^{76}$

Venous access is a crucial issue in the management of children undergoing ITI, as well as in those on primary prophylaxis. Although peripheral venipuncture is the first choice, CVADs are often necessary for these young children, especially for high-dose regimens. Totally implantable catheters (ports) are preferred to external CVADs because of the lower risk of complications, especially infection and thrombosis. However, CVADs should not be used routinely in children and, if required, should be removed as soon as feasible. ${ }^{85,86}$ Indeed, the development of catheter infections may cause dangerous interruptions to prophylaxis, and in particular to ITI. This may prolong time taken to achieve successful tolerance or lead to treatment failure by provoking an anamnestic increase in the inhibitor titer. ${ }^{76}$ Arteriovenous fistulae could be considered a promising option for children aged one year and older who have experienced CVAD failure. Indeed, positive results have been reported in two studies conducted in Italy and the US, respectively. ${ }^{87,88}$ However, this approach requires a highly experienced surgeon and continuous follow-up, so should be performed only in specialized centers.

\section{Comprehensive care of hemophilic patients}

Hemophilia care does not consist only of replacement therapy and hematologic follow-up. The hematologist's clinical and laboratory expertise should be conjugated to other diagnostic and therapeutic facilities for the management of bleeding at various sites, surgery, and chronic complications. The need for a multidisciplinary integrated approach at specialized centers for this rare congenital disease requiring complex management has been recognized since the 1960s..$^{89,90}$

Comprehensive care of hemophilic patients addresses treatment and prevention of bleeding, long-term management of hemophilic arthropathy and other complications of bleeding, management of significant complications of treatment (development of inhibitors and transfusion-transmitted infections), and the psychosocial support and education required to manage the bleeding disorder, including venous access and home treatment. Therefore, the core team at a hemophilia comprehensive treatment center (HCTC), including hematologists, laboratory staff, nurses, physiotherapists and social workers, should involve the collaboration of several specialists. Genetic counseling for patients and their families, 
and advice about prenatal diagnosis and management of pregnancy and delivery in hemophilia carriers should be offered. Joint disease is now minimal in younger patients on primary or early secondary prophylaxis, but the impact on adult patients is still significant, and likely to increase as the life expectancy of hemophilic patients continues to increase. Thus, a trained orthopedic surgery team should be available, at least at major HCTCs. Another crucial issue for patients born before the mid-1980s is the management of chronic hepatitis $\mathrm{B}$ and $\mathrm{C}$ infection and the related long-term complications, and of human immunodeficiency virus infection, in particular after the advent of highly active anti-retroviral therapy. This requires close interaction with hepatologists and infectious disease specialists. Moreover, new challenges are emerging in the care of aging patients, not only in terms of the comorbidities typically associated with hemophilia, but also with regard to common age-related illnesses, such as cardiovascular disease and cancer. ${ }^{91}$

The delivery of a high-quality comprehensive service to patients with bleeding disorders has been modeled upon defined standards and a network of designated HCTCs in the UK, US, and other Western countries. ${ }^{89,92}$ These principles have been summarized recently by the European Association for Haemophilia and Associated Disorders. ${ }^{92}$ In developing countries, despite a shortage or absence of treatment products, the development of local expertise thanks to twinning programs is resulting in an improved outlook and reduction in mortality. ${ }^{89,90}$ Interestingly, this finding is also true for developed countries; in the US, patients who received their care in HCTCs had lower hospital admission and mortality rates than those who accessed their care outside. ${ }^{93}$ These data highlight the importance of ongoing specialist services for this group of patients.

\section{Conclusion and perspectives}

Since the advent of modern replacement treatment in the early 1970s, hemophilia has become an example of successful management of a congenital chronic disease. This has been associated with an impressive increase in patient life expectancy, which was previously less than 30 years. In parallel, the increasing implementation of prophylaxis in children has enabled avoidance of or significant reduction in the crippling consequences of hemophilic arthropathy. The development of effective viral inactivation techniques, together with methods used to screen viruses in blood donations and plasma pools, has greatly improved the safety of plasma-derived products, confirmed by the fact that no blood-borne transmission of hepatitis viruses or human immunodeficiency virus has occurred in the last 15 years. ${ }^{5}$ However, the introduction of recombinant products has contributed greatly to the perceived increased safety of replacement therapy, and to the more widespread use of prophylaxis and home treatment. Because of uncertainties with regard to as yet unknown pathogens and the potential impact of prion disease in the hemophilic population reliant on blood derivatives,${ }^{94}$ there has been a strong push in some Western countries to treat hemophilic children only with rFVIII products, as recommended by several national guidelines. ${ }^{2,95}$ Furthermore, with safety as a priority, the manufacturing process for $\mathrm{rFVIII}$ products has further evolved during the last few years to minimize the risk of pathogen transmission, with the improvement of protein purification techniques and viral inactivation steps, and avoidance of human or animal proteins at any stage of their manufacture. ${ }^{96}$

Prophylactic therapy beginning at an early age should be considered the evidence-based treatment of choice for children with severe hemophilia, enabling normal physical, psychologic, and social development. The optimal regimen is still debated. From an analysis of data in the recent literature it is clear that the intensity and duration of prophylaxis should be individualized on the basis of the patient's clinical needs, particularly with respect to venous access. Recent studies also suggest a role for delayed secondary prophylaxis in increasing joint protection compared with on-demand therapy. An undoubtedly favorable impact of prophylaxis is detectable at any age. In this respect, the individualization of regimens may help to devise more cost-effective treatments. It is noteworthy that assessment of outcome in hemophilia requires longer follow-up, should not be confined to direct costs of concentrates, and should take into account all the additional health resources related to the management of hemophilia and its orthopedic complications. ${ }^{97}$ Moreover, newer outcome measures useful in determining the overall benefits to patients should be added to the clinical outcomes, including Health Related Quality of Life (HRQoL) assessment. ${ }^{97}$ Generic and validated disease-specific HRQoL questionnaires help to assess and quantify the multidimensional perception of well-being, including physical and psychologic components. ${ }^{98}$

The universal implementation of primary prophylaxis in children remains impractical, particularly in developing countries where only resources for minimal on-demand treatment are available. However, a series of barriers still needs to be overcome even in Western countries, including venous access, long-term adherence to treatment, and psychosocial problems. 
The development of inhibitors is presently the most serious complication of factor concentrate replacement therapy. Although it is the result of a complex interaction between multiple genetic and environmental factors, the exact impact of single factors, in particular the type of concentrate, remains to be established. Recent studies have suggested that prophylaxis could exert a protective effect on inhibitor risk. A $60 \%-80 \%$ reduction of inhibitor development could be expected according to the available data. This possibility will have striking implications from the clinical and pharmacoeconomic points of view in terms of cost-effectiveness of prophylaxis and reduction in the highest burden of costs for management of patients with inhibitors.

The excellent results in patients without inhibitors are leading to prophylaxis approaches using both bypassing agents (rFVIIa and APCC) in children with an inhibitor because these patients are at high risk of developing severe musculoskeletal impairment and life-threatening bleeds. However, larger and more rigorous studies are needed to confirm that extrapolations from patients without inhibitors are applicable to those with inhibitors. Despite uncertainties concerning optimal treatment regimens, ITI should be the treatment of choice for children with an inhibitor, starting when the inhibitor titer is low and with the availability of stable and long-lasting venous access.

Hemophilia A and B have been extensively researched in the field of gene therapy because they represent monogenic disorders for which animal models are available, the threshold for success is relatively low (even gene expression at low levels of $2 \%-5 \%$ may result in significant modification of clinical phenotype), and the outcome may be easily evaluated by measuring plasma factor levels. Between 1998 and 2001, five Phase I clinical trials were carried out using in vivo gene transfer by different gene delivery systems (retroviral, adenoviral, or adeno-associated viral vectors as well as nonviral methods). ${ }^{99}$ These studies provided important safety data for the various strategies used. However, at variance with animal studies, none of them achieved long-term expression of the clotting factor at therapeutic levels. Human-specific challenges to gene transfer have been shown, particulary related to the immune response either against the vector or against the transgene product. Therefore, taking a step backwards to animal and cellular models, research is now aimed at finding new strategies for improving transduction efficacy, identifying the best target tissue (liver or muscle), and achieving long-term expression in the absence of an immune response. ${ }^{99}$

In conclusion, although a cure through gene therapy is far from being implemented, hemophilia is currently the most
Table 6 Strategies for bioengineered factor concentrates with modified properties in hemophilia

\begin{tabular}{ll}
\hline Target & Strategies \\
\hline Increased efficiency of production & $\begin{array}{l}\text { Improved secretion efficiency } \\
\text { Higher mRNA stability, facilitated } \\
\text { intracellular transport* }\end{array}$ \\
Increased potency and stability & $\begin{array}{l}\text { Active site modifications* } \\
\text { FVIlla stabilization }\end{array}$ \\
Prolonged half-life & Pegylated liposomes (FVIII) \\
& Pegylation (FVIII, FIX, FVIla) \\
& Polysialylation (FVIII) \\
& Fc fragment fusion (FIX) \\
& Albumin fusion (FVIIa) \\
& Porcine/human hybrids \\
Reduced immunogenicity & CI/C2 domain and inhibitor epitope \\
& mapping-driven modifications*
\end{tabular}

Notes: "On development by basic studies; **Phase II clinical trial ongoing.

effectively and safely treated monogenic inherited disorder. ${ }^{5}$ However, this statement applies only to patients living in Europe, the US, and in a few high-income Asian countries, where highly safe antihemophilic products are widely available and comprehensive care is provided. The main goal for the future is to address the lack of satisfactory hemophilia care worldwide for more than two-thirds of people with hemophilia. Unfortunately, the evolution and increasing availability of products have not been matched by a decrease in their costs. Further encouraging perspectives come from new factor concentrates with longer half-lives or other modifications presently in clinical trials ${ }^{100}$ or in development ${ }^{99}$ (Table 6). These genetically engineered products will enable us to reduce the impact of some crucial unsolved issues, like the need for frequent infusions and for CVADs in children, facilitating the implementation of prophylaxis or ITI, the development of inhibitors and, probably, the consumption of replacement factors and costs of treatment.

\section{Disclosure}

The authors declare no conflicts of interest.

\section{References}

1. Mannucci PM, Tuddenham EG. The hemophilias - from royal genes to gene therapy. N Engl J Med. 2001;344(5):1773-1779.

2. van Dijk K, Fischer K, van der Bom JG, Grobbee DE, van den Berg HM. Variability in clinical phenotype of severe haemophilia: The role of the first joint bleed. Haemophilia. 2005;11(5):438-443.

3. Santagostino E, Mannucci PM, for the Italian Association of Haemophilia Centres (AICE). Guidelines on replacement therapy for haemophilia and inherited coagulation disorders in Italy. Haemophilia. 2000;6(1):1-10.

4. Rosendaal G, Lafeber FP. Pathogenesis of hemophilic arthropathy. Haemophilia. 2006;12 Suppl 3:117-121.

5. Mannucci PM. Back to the future: A recent history of hemophilia treatment. Haemophilia. 2008;14 Suppl 3:10-18. 
6. Plug I, van der Bom JG, Peters M, et al. Thirty years of hemophilia treatment in the Netherlands, 1972-2001. Blood. 2004;104(12):3494-3500.

7. Franchini M, Coppola A, Molinari AC, et al. Forum on the role of recombinant factor VIII in children with severe haemophilia A. Haemophilia. 2009;15(2):578-586.

8. Wight J, Paisley S. The epidemiology of inhibitors in haemophilia A: A systematic review. Haemophilia. 2003;9(4):418-435.

9. Street AM, Ljung R, Lavery SA. Management of carriers and babies with haemophilia. Haemophilia. 2008;14 Suppl 3:181-187.

10. Ljung R, Petrini P, Nilsson IM. Diagnostic symptoms of severe and moderate haemophilia A and B. A survey of 140 cases. Acta Paediatr Scand. 1990;79(2):196-200.

11. Kulkarni R, Lusher JM, Henry RC, Kallen DJ. Current practices regarding newborn intracranial haemorrhage and obstetrical care and mode of delivery of pregnant haemophilia carriers: A survey of obstetricians, neonatologists and haematologists in the United States, on behalf of the National Hemophilia Foundation's Medical and Scientifical Advisory Council. Haemophilia. 1999;5(6): 410-415.

12. van den Berg HM, de Groot PHG, Fischer K. Phenotypic heterogeneity in severe hemophilia. J Thromb Haemost. 2007;5 Suppl 1:151-156.

13. Aledort L, Haschmeyer RH, Pettersson H. A longitudinal study of orthopedic outcomes for severe factor-VIII-deficient hemophiliacs. The Orthopedic Outcome Study Group. J Intern Med. 1994; 236(4):391-399.

14. Gilbert MS. Prophylaxis: Musculoskeletal evaluation. Semin Hematol. 1993;3 Suppl 2:3-6.

15. Pettersson H, Ahlberg A, Nilsson IM. A radiologic classification of hemophilic arthropathy. Clin Orthop Relat Res. 1980;(149):153-159.

16. Fischer K, van der Bom JG, Mauser-Bunschoten EP, et al. The effects of postponing prophylactic treatment on long-term outcome in patients with severe hemophilia. Blood. 2002;99(7):2337-2341.

17. Ahlberg A. Haemophilia in Sweden. VII. Incidence, treatment and prophylaxis of arthropathy and other musculoskeletal manifestations of hemophilia A and B. Acta Orthop Scand Suppl. 1965;Suppl 77: 3-132.

18. Nilsson IM, Berntorp E, Löfqvist T, Pettersson H. Twenty-five years experience of prophylactic treatment in severe haemophilia A and B J Intern Med. 1992;232(1):25-32.

19. Astermark J, Petrini P, Tengborn L, Schulman S, Ljung R, Berntorp E. Primary prophylaxis in severe haemophilia should be started at an early age but can be individualized. Br J Haematol. 1999;105(4): 1109-1113.

20. Kreuz W, Escuriola-Ettingshausen C, Funk M, et al. When should prophylactic treatment in patients with hemophilia A and B start? The German experience. Haemophilia. 1998;4(4):413-417.

21. Panicker J, Warrier I, Lusher J. The impact of prophylaxis in children with severe hemophilia. Haemophilia. 2002;8(4):489-490.

22. Yee TT, Beeton K, Griffioen A, et al. Experience of prophylaxis treatment in children with severe hemophilia. Haemophilia. 2002; $8(2): 76-82$.

23. van den Berg HM, Fischer K, Mauser-Bunschoten EP, et al. Long-term outcome of individualized prophylactic treatment of children with severe haemophilia. Br J Haematol. 2001;112(3):561-565.

24. Berntorp E, Astermark J, Bjorkman S, et al. Consensus perspectives on prophylaxis therapy for haemophilia: Summary statement. Haemophilia. 2003;9 Suppl 1:1-4.

25. Donadel-Claeyssens S. Current co-ordinated activities of the PEDNET (European Paediatric Network for Haemophilia Management). Haemophilia. 2006;12(2):124-127.

26. Valentino LA. Secondary prophylaxis therapy: What are benefits, limitations and unknown? Haemophilia. 2004;10(2):147-157.

27. Stobart K, Iorio A, Wu JK. Clotting factor concentrates given to prevent bleeding and bleeding-related complications in people with hemophilia A or B. Cochrane Database Syst Rev. 2006;2:CD003429.
28. Manco-Johnson MJ, Abshire TC, Shapiro AD, et al. Prophylaxis versus episodic treatment to prevent joint disease in boys with severe hemophilia. N Engl J Med. 2007;357(6):535-544.

29. Doria AS, Lundin B, Kilcoyne RF, et al. Reliability of progressive and additive MRI scoring systems for evaluation of haemophilic arthropathy in children: Expert MRI Working Group of the International Prophylaxis Study Group. Haemophilia. 2005;11(3):245-253.

30. Gringeri A, Lundin B, von Mackensen S, et al. Primary and secondary prophylaxis in children with haemophilia A reduces bleeding frequency and arthropathy development compared to on-demand treatment: A 10-year, randomized, clinical trial. J Thromb Haemost. 2009;7 Suppl 2:Abstr OC-MO-034.

31. Berntorp E, Boulyjenkov V, Brettler D, et al. Modern treatment of haemophilia. Bull World Health Organ. 1995;73(5):691-701.

32. Fischer K, Astermark J, van der Bom JG, et al. Prophylactic treatment for severe haemophilia: Comparison of an intermediate-dose to a high-dose regimen. Haemophilia. 2002;8(6):753-760.

33. Petrini P. Identifying and overcoming barriers to prophylaxis in the management of haemophilia. Haemophilia. 2007;13 Suppl 2:16-22.

34. Petrini P. What factors should influence the dosage and interval of prophylactic treatment in patients with severe haemophilia A and B? Haemophilia. 2001;7(1):99-102.

35. Feldman BN, Pai M, Rivard GE, et al. Tailored prophylaxis in severe hemophilia A: Interim results from the first 5 years of the Canadian Hemophilia Primary Prophylaxis Study. J Thromb Haemost. 2006;4(6):1228-1236.

36. Geraghty S, Dunkley T, Harrington C, et al. Practice patterns in haemophilia A therapy - global progress towards optimal care. Haemophilia. 2006;12(1):75-81.

37. Srivastava A, Giangrande P, Poon MC, et al. Guidelines for the Management of Hemophilia. World Federation of Hemophilia, 2005. Available at http://www.wfh.org/2/docs/Publications/Diagnosis_and_Treatment/Guidelines_Mng_Hemophilia.pdf. Last accessed on Jan 20, 2010.

38. Medical and Scientific Advisory Council. MASAC recommendation concerning prophylaxis (regular administration of clotting factor concentrate to prevent bleeding). Recommendation 170. New York, NY: National Hemophilia Foundation; 2006. Available at: http://www.hemophilia.org/NHFWeb/MainPgs/MainNHF.aspx menuid $=57 \&$ contentid=582 Last accessed on Jan 20, 2010 .

39. Medical and Scientific Advisory Council. MASAC recommendation concerning prophylaxis (regular administration of clotting factor concentrate to prevent bleeding). Recommendation 179. New York, NY: National Hemophilia Foundation; 2007. Available at: http://www.hemophilia. org/NHFWeb/MainPgs/MainNHF.aspx?menuid=57\&contentid=1007. Last accessed on Jan 20, 2010.

40. Fischer K, van der Bom JG, Prejs R, et al. Discontinuation of prophylactic therapy in severe haemophilia: Incidence and effects on outcome. Haemophilia. 2001;7(6):544-550.

41. van Dijk K, Fischer K, van der Bom JG, Scheibel E, Ingerslev J, van den Berg HM. Can long-term prophylaxis for severe haemophilia be stopped in adulthood? Results from Denmark and the Netherlands. Br J Haematol. 2005;130(1):107-112.

42. Brackmann HH, Eickhoff HJ, Oldenburg J, Hammerstein U. Longterm therapy and on-demand treatment of children and adolescents with severe hemophilia A: 12 years of experience. Haemostasis. 1992;22(5):251-258

43. Manco-Johnson MJ, Nuss R, Geraghty S, et al. Results of secondary prophylaxis in children with severe hemophilia. Am J Hematol. 1994;47(2):113-117.

44. Liesner RJ, Khair K, Hann IM. The impact of prophyactic treatment on children with severe hemophilia. Br J Haematol. 1996;92(4):973-978.

45. Cohen A, White E, Bernstein C, et al. Effects of secondary prophylaxis in hemophilia children with frequent hemarthrosis. Transfusion. 1997;37(5):534-535. 
46. Miners AH, Sabin CA, Tolley KH, Lee CA. Assessing the effectiveness and cost-effectiveness of prophylaxis against bleeding in patients with severe haemophilia and severe von Willebrand's disease. J Intern Med. 1998;244(6):515-522.

47. Fischer K, Van Dijk K, Van den Berg HM. Late prophylaxis for severe hemophilia: Effects of prophylaxis started in adulthood. J Thromb Haemost. 2005;3 Suppl 1:Abstr OR205.

48. Tagliaferri A, Franchini M, Coppola A, et al. Effects of secondary prophylaxis started in young adult hemophiliacs. Haemophilia. 2008;14(5):945-951.

49. Coppola A, Franchini M, Tagliaferri A. Prophylaxis in people with haemophilia. Thromb Haemost. 2009;101(4):674-681.

50. Wight J, Paisley S. The epidemiology of inhibitors in haemophilia A. A systematic review. Haemophilia. 2003;9(4):418-435.

51. Mehta R, Parameswaran R, Shapiro AD. An overview of the history, clinical practice concerns, comparative studies and strategies to optimize therapy of bypassing agents. Haemophilia. 2006;12 Suppl 6:54-61.

52. Rodriguez-Merchan EC, Hedner U, Heijnen L, et al. Prevention of haemophilic arthropathy during childhood. May common orthopaedic management be extrapolated from patients without inhibitors to patients with inhibitors? Haemophilia. 2008;14 Suppl 6:68-81.

53. UK Haemophilia Centre Doctors' Organization. The incidence of factor VIII and factor IX inhibitors in the hemophilia population of the UK and their effect on subsequent mortality, 1977-99. J Thromb Haemost. 2004;2(7):1047-1054.

54. Gringeri A, Mantovani LG, Scalone L, Mannucci PM. Cost of care and quality of life for patients with hemophilia complicated by inhibitors: The COCIS Study Group. Blood. 2003;102(7):2358-2363.

55. Scalone L, Mantovani LG, Mannucci PM, Gringeri A. Quality of life is associated to the orthopaedic status in haemophilic patients with inhibitors. Haemophilia. 2006;12(2):154-162.

56. Coppola A, Santoro C, Tagliaferri A, Franchini M, Di Minno G. Understanding inhibitor development in haemophilia A: Towards clinical prediction and prevention strategies. Haemophila. 2010;16 Suppl 1:13-19.

57. Schwaab R, Brackmann HH, Meyer C, et al. Haemophilia A: Mutation type determines the risk of inhibitor formation. Thromb Haemost. 1995;74(6):1402-1406.

58. Astermark J, Oldenburg J, Pavlova A, Berntorp E, Lefvert AK; MIBS Study Group. Polymorphisms in the IL10 but not in the IL1 beta and IL4 genes are associated with inhibitor development in patients with hemophilia A. Blood. 2006;107(8):3167-3172.

59. Astermark J, Oldenburg J, Carlson J, et al. Polymorphisms in the TNFA gene and risk of inhibitor development in patients with hemophilia A. Blood. 2006;108(12):3739-3745.

60. Astermark J, Wang X, Oldenburg J, Berntorp E, Lefvert AK; MIBS Study Group. Polymorphisms in the CTLA-4 gene and inhibitor development in patients with severe hemophilia A. JThromb Haemost. 2007;5(2):263-265.

61. Matzinger P. The danger model: A renewed sense of self. Science. 2002;8(5566):76-82.

62. Gouw SC, van der Bom JG, van den Berg HM. Treatment-related risk factors of inhibitor development in previously untreated patients with hemophilia A: The CANAL cohort study. Blood. 2007;109:4648-4654.

63. Gouw SC, van den Berg HM, le Cessie S, van der Bom JG. Treatment characteristics and the risk of inhibitor development: A multi-center cohort study among previously untreated patients with severe hemophilia A. J Thromb Haemost. 2007;5(7):1383-1390.

64. Morado M, Villar A, Jiménez-Yuste V, Quintana M, Hernandez Navarro F. Prophylactic treatment effects on inhibitor risk: Experience in one centre. Haemophilia. 2005;11(2):79-83.

65. Santagostino E, Mancuso ME, Rocino A, et al. Environmental risk factors for inhibitor development in children with haemophilia A: A case-control study. Br J Haematol. 2005;130(3):422-427.
66. Kurnik K, Bidlingmaier C, Engl W, Chehadeh H, Reipert B, Auerswald G. New early prophylaxis regimen that avoids immunological danger signals can reduce FVIII inhibitor development. Haemophilia. Oct 29, 2009. [Epub ahead of print]

67. Gouw SC, van der Bom JG, Auerswald G, Ettinghausen CE, Tedgård U, van der Berg HM. Recombinant versus plasma-derived factor VIII products and the development of inhibitors in previously untreated patients with severe hemophilia A: The CANAL cohort study. Blood. 2007;109(11):4693-4697.

68. Goudemand J, Rothschild C, Demiguel V, et al; FVIII-LFB and recombinant FVIII study groups. Influence of the type of factor VIII concentrate on the incidence of factor VIII inhibitors in previously untreated patients with severe hemophilia A. Blood. 2006;107(1):46-51.

69. Chalmers EA, Brown SA, Keeling D, et al; Paediatric Working Party of UKHCDO. Early factor VIII exposure and subsequent inhibitor development in children with severe haemophilia A. Haemophilia. 2007;13(2):149-155.

70. Peerlinck K, Hermans C. Epidemiology of inhibitor formation with recombinant factor VIII replacement therapy. Haemophilia. 2006;12(6):579-590.

71. Escuriola-Ettingshausen C, Kreuz W. Recombinant vs plasma-derived products, especially those with intact VWF, regarding inhibitor development. Haemophilia. 2006;12 Suppl 6:102-106.

72. Paisley S, Wight J, Currie E, Knight J. The management of inhibitors in haemophilia A: Introduction and systematic review of current practice. Haemophilia. 2003;9(4):405-417.

73. Berntorp E, Shapiro A, Astermark J, et al. Inhibitor treatment in haemophilias A and B: Summary statement for the 2006 international consensus conference. Haemophilia. 2006;12 Suppl 6:1-7.

74. Hay CR, Baglin TP, Collins PW, Hill FG, Keeling DM. The diagnosis and management of factor VIII and IX inhibitors: A guideline from the UK Haemophilia Centre Doctors Organization (UKHCDO). Br J Haematol. 2000;111(1):78-90.

75. Gringeri A, Mannucci PM, for the Italian Association of Haemophilia Centres. Italian guidelines for the diagnosis and treatment of patients with haemophilia and inhibitors. Haemophilia. 2005;11(6):611-619.

76. DiMichele D, Hoots WK, Pipe SW, Rivard GE, Santagostino E. International workshop on immune tolerance induction: Consensus recommendations. Haemophilia. 2007;13 Suppl 1:1-22.

77. Wight J, Paisley S, Knight C. Immune tolerance induction in patients with haemophilia A with inhibitors. A systematic review. Haemophilia. 2003;9(4):436-463.

78. Mariani G, Ghirardini A, Bellocco R. Immune tolerance in hemophiliaprincipal results from the International Registry. Thromb Haemost. 1994;72(1):155-158.

79. DiMichele D, Kroner BL. The North American Immune Tolerance Registry: Practices, outcomes, outcome predictors. Thromb Haemost. 2002;87(1):52-57.

80. Lenk H. The German Registry of immune tolerance treatment in hemophilia - 1999 update. Haematologica. 2000;10 Suppl:45-47.

81. Kroner BL. Comparison of the international immune tolerance registry and the North American immune tolerance registry. Vox Sang. 1999;77 Suppl 1:33-37.

82. Coppola A, Margaglione M, Santagostino E, et al. FVIII gene (F8) mutations as predictors of outcome in immune tolerance induction of hemophilia A patients with high-responding inhibitors. J Thromb Haemost. 2009;7(11):1809-1815.

83. DiMichele DM, Hay CR. The international immune tolerance study: A multicenter prospective randomized trial in progress. $J$ Thromb Haemost. 2006;4(10):2271-2273.

84. Gringeri A. VWF/FVIII concentrates in high-risk immunotolerance: The RESIST study. Haemophilia. 2007;13 Suppl 5:73-77.

85. Komvilaisak P, Connolly B, Naqvi A, Blanchette V. Overview of the use of implantable venous access devices in the management of children with inherited bleeding disorders. Haemophilia. 2006;12 Suppl6: $87-93$. 
86. Ljung R. The risk associated with indwelling catheters in children with hemophilia. Br J Haematol. 2007;138(5):580-586.

87. McCarthy WJ, Valentino LA, Bonilla AS, et al. Arteriovenous fistula for long-term venous access for boys with hemophilia. J Vasc Surg. 2007;45(5):986-990.

88. Mancuso M, Berardinelli L, Beretta C, Raiteri M, Pozzoli E, Santagostino E. Improved treatment feasibility in children with hemophilia using arteriovenous fistulae: The results after seven years of follow-up. Haematologica. 2009;94(5):687-692.

89. Bolton-Maggs P. Optimal haemophilia care versus the reality. $\mathrm{Br} \mathrm{J}$ Haematol. 2005;132(6):671-682.

90. Evatt BL, Black C, Batorova A, Street A, Srivastava A. Comprehensive care for haemophilia around the world. Haemophilia. 2004;10 Suppl 4: 9-13.

91. Mannucci PM, Schutgens REG, Santagostino E, Mauser-Bunschoten EP. How I treat age-related morbidities in elderly persons with hemophilia. Blood. 2009; 114(26):5256-5263.

92. Colvin BT, Astermark J, Fischer K, et al. European principles of haemophilia care. Haemophilia. 2008;14(2):361-374.

93. Soucie JM, Nuss R, Evatt B, et al. Mortality among males with hemophilia: Relations with the source of medical care. Blood. 2000;96(2): 437-442.
94. Llewelyn CA, Hewitt PE, Knight RS, et al. Possible transmission of variant Creutzfeldt-Jakob disease by blood transfusion. Lancet. 2004;363(9407):417-421.

95. Keeling D, Tait C, Makris M. Guideline on the selection and use of therapeutic products to treat haemophilia and other hereditary bleeding disorders. Haemophilia. 2008;14(4):671-684.

96. Pipe SW. Recombinant clotting factors. Thromb Haemost. 2008; 99(5):840-850.

97. Bohn RL, Schramm W, Bullinger M, et al. Outcome measures in hemophilia: More than just factor levels. Haemophilia. 2004;10 Suppl 1:2-8.

98. Gringeri A, von Mackensen S. Quality of life in haemophilia. Haemophilia. 2008;14 Suppl 3:19-25.

99. Pipe SW, High KA, Ohashi K, Ural AU, Lillicrap D. Progress in the molecular biology of inherited bleeding disorders. Haemophilia. 2008; 14 Suppl 3:130-137.

100. Spira J, Plyushch OP, Andreeva TA, Andreev Y. Prolonged bleedingfree period following prophylactic infusion of recombinant FVIII reconstituted with pegylated liposomes. Blood. 2006:108(12) $3668-3673$.
Journal of Blood Medicine

\section{Publish your work in this journal}

The Journal of Blood Medicine is an international, peer-reviewed, open access, online journal publishing laboratory, experimental and clinical aspects of all topics pertaining to blood based medicine including but not limited to: Transfusion Medicine; Blood collection, Donor issues, Transmittable diseases, and Blood banking logistics; Immunohematology; Artificial and alternative

\section{Dovepress}

blood based therapeutics; Hematology; Biotechnology/nanotechnology of blood related medicine; Legal aspects of blood medicine; Historical perspectives. The manuscript management system is completely online and includes a very quick and fair peer-review system. Visit http://www.dovepress.com/ testimonials.php to read real quotes from published authors.

Submit your manuscript here: http://www.dovepress.com/Journal-of-blood-medicine-journal 